\title{
Suppression of multiple scattering for the critical mixture polystyrene/ cyclohexane: Application of the one-beam cross correlation technique
}

\author{
Jörg-Michael Schröder and Alfons Becker \\ Max-Planck-Institut für Polymerforschung, Ackermannweg 10, D-55128 Mainz, Germany \\ Simone Wiegand ${ }^{\text {a) }}$ \\ Max-Planck-Institut für Polymerforschung, Ackermannweg 10, D-55128 Mainz, Germany \\ and Forschungszentrum Jülich GmbH, IFF-Weiche Materie, D-52428 Jülich, Germany
}

(Received 16 September 2002; accepted 25 March 2003)

\begin{abstract}
Multiple scattering becomes a problem close to the critical point, especially in systems with a large difference in the refractive index and large correlation length amplitudes as for the case of polystyrene/cyclohexane. In this work we demonstrate the application of the one-beam cross correlation technique and show that in the reduced temperature range of $t_{\text {red }}=1.66 \times 10^{-5}-0.03$ the multiple scattering is successfully suppressed. Furthermore, we measured the angular dependent static scattering intensity. By using the amplitude of the cross correlation function we obtained the correct singly scattered intensities. Those corrected intensities can be analyzed in the framework of the Ornstein-Zernike plot to obtain the correlation length $\xi$. The analysis of the obtained static data is in good agreement with the dynamic data. Limitations for temperature and angular dependent measurements in the Ornstein-Zernike plot close to $T_{c}$ are discussed in detail. (C) 2003 American Institute of Physics. [DOI: 10.1063/1.1574799]
\end{abstract}

\section{INTRODUCTION}

Dynamic and static light scattering are commonly used methods to study structural and dynamic properties of polymer solutions, colloidal suspensions or more general multicomponent systems. Also the critical behavior of static and transport properties in fluids and fluid mixtures has been studied frequently. The critical slowing down of the order parameter fluctuations can be detected by measuring the time dependent correlation function of the scattered photons, while an increase of the concentration fluctuations manifests itself by an increase of the scattering intensity. However, the analysis of these experiments is restricted to transparent samples in order to avoid multiple scattering. This limits the accessible temperature range in critical solutions by conventional light scattering methods.

In the past three decades these order parameter fluctuations which result in the so-called critical opalescence have been studied in binary mixtures and polymer solutions. ${ }^{1}$ The divergence of the correlation length is described by the asymptotic power law $\xi=\xi_{0} t_{\text {red }}^{-\nu}$, with the reduced temperature $t_{\text {red }}=\left(T-T_{c}\right) / T_{c}$ and the critical exponent $\nu=0.63$ of the three-dimensional (3D) Ising model. It is generally accepted that all fluid systems belong to the same universality class of the 3D-Ising model characterized by a set of critical exponents. ${ }^{2}$ For some ionic liquid mixtures, deviations from the Ising behavior result in the manifestation of in a crossover theory. ${ }^{3,4}$

Close to the critical point the interpretation of the light scattering results is complicated by multiple scattering. Recent measurements on polydisperse polymer solutions of

\footnotetext{
${ }^{a)}$ Electronic mail: s.wiegand@fz-juelich.de; http://www.fz-juelich.de/iff/ personen/S.Wiegand/
}

polystyrene in cyclohexane and methylcyclohexane have been reported ${ }^{5}$ which, however, could only be analyzed for reduced temperatures $t_{\text {red }} \geqslant 3.4 \times 10^{-4}$ due to the presence of multiple scattering closer to the critical point.

Several attempts have been made to determine the amount of multiply scattered light theoretically ${ }^{6-8}$ or by Monte Carlo simulations. ${ }^{9}$ The theoretical method by Shanks and Sengers ${ }^{6}$ calculates the double-scattering contributions in critically opalescent samples for which the angle dependent scattering cross section is given by the OrnsteinZernike equation. An extension of the theory to higher orders of multiple scattering becomes very complicated due to manifold integrals. Under the conditions of multiple scattering a direct simulation of the scattering intensity by Monte Carlo simulations seems to be a more promising approach. ${ }^{9}$ Unfortunately, the theoretical and simulation approaches require a precise knowledge of the experimental constraints which are often not accessible in the necessary accuracy or are result of the measurement.

In recent years several experimental techniques such as the two-color set-up, ${ }^{10,11}$ the $3 \mathrm{D}$ set-up, ${ }^{12-14}$ and lately also the one-beam set-up, ${ }^{15}$ have been used to suppress multiple scattering contributions in dynamic light scattering experiments. The geometries of the different set-ups are chosen in such a way, that the only singly scattered light contributes to the recorded cross correlation function. Lately it was demonstrated that all three techniques can be used to obtain the correct static light scattering information in highly concentrated suspensions of latex spheres. ${ }^{12,16,17}$ The basic idea of the so-called two-color coding experiment and the threedimensional set-up is similar. ${ }^{18}$ In $3 \mathrm{D}$ and two-color set-up the scattering experiment is done twice in the same scattering volume with identical resulting scattering vectors $q_{1,2}$ 
$=\left(4 \pi n_{1,2} / \lambda_{1,2}\right) \sin \left(\theta_{1,2} / 2\right)$ with $n$ and $\lambda$ denoting the refractive index and the wavelength, respectively. An excellent review on the different experimental approaches and results was recently published by Pusey. ${ }^{19}$

In this work we use a one-beam set-up ${ }^{15,20,21}$ which operates on a different principle. The method uses the fact that the singly scattered light results in a larger coherence area compared to the multiple scattered light. By optimizing the distance between the two detectors so that they are still within the coherence area of the singly scattered light but not within the coherence area of the multiply scattered light. This way the singly scattered light is correlated, while the multiply scattered light due to a smaller coherence area is no longer correlated. This allows the correct determination of the static and dynamic scattering properties. Lately the onebeam cross correlation technique was successfully applied to depolarized scattering experiments, which are very sensitive to effects of multiple scattering. ${ }^{22}$

Recently, we used the $3 \mathrm{D}$ cross correlation method to study the temperature dependent critical fluctuations of the local composition of a solution of polystyrene in cyclohexane. ${ }^{23}$ We investigate a temperature range of $20 \mathrm{~K}$ down to $2 \times 10^{-3} \mathrm{~K}$ above $T_{c}$ which corresponds to reduced temperature range of $t_{\text {red }}$ of $6.8 \times 10^{-6}$ to 0.067 with $T_{c}$ $=293.492 \mathrm{~K}$. We determined the intensity of the singly scattered light at a scattering angle $\theta=90^{\circ}$ and showed that it is perfectly described by the Ornstein-Zernike function in the entire temperature range. Even very close to the critical point, where the turbidity reaches a value of $2.4 \mathrm{~cm}^{-1}$, no deviations are noticeable. In addition, we compared the amount of singly and multiply scattered light with Monte Carlo simulation of the multiple scattering processes and found a good agreement.

In this paper we apply the one-beam cross correlation technique to the same polystyrene/cyclohexane sample investigated in the previous paper. Additionally to the measurements at scattering angle of $\theta=90^{\circ}$ we perform also angular dependent measurements in the range of $\theta=40^{\circ}-140^{\circ}$. Using the amplitude of the cross correlation function we determine the singly scattered light intensity which we analyzed using the Ornstein-Zernike function. We compare the results determined with the one-beam set-up with those obtained with the $3 \mathrm{D}$ cross correlation technique.

\section{THEORY AND DATA ANALYSIS}

\section{A. One-beam cross correlation technique}

The normalized cross correlation function of the scattered light intensity $g^{(2)}(t)$ is given by

$$
g^{(2)}(t)=1+\beta\left|g^{(1)}(t)\right|^{2},
$$

where $g^{(1)}(t)$ is the normalized field correlation function of the singly scattered light and $\beta$ denotes the amplitude of the cross correlation function, which is proportional to the intensity ratio of the singly scattered light to the totally scattered light including the multiple scattering contributions. In this way the intensity of the singly scattered light $\left\langle i_{s}\right\rangle$ can be obtained from the totally scattered light intensity $\left\langle i_{\text {tot }}\right\rangle$

$$
\left\langle i_{s}\right\rangle \propto \beta\left\langle i_{\text {tot }}\right\rangle .
$$

\section{B. Scattering behavior of critical binary mixture}

The single scattering intensity $\left\langle i_{s, \theta}\right\rangle$ of a critical binary mixture can be analyzed in the frame work of the OrnsteinZernike theory. ${ }^{24}$ According to this theory $\left\langle i_{s, \theta}\right\rangle$ can be written in the form

$$
\left\langle i_{s, \theta}\right\rangle=A\left(1+t_{\text {red }}\right) \frac{t_{\text {red }}-(2-\eta) \nu}{1+\left(q \xi_{0} t_{\text {red }}{ }^{-\nu}\right)^{2}} .
$$

We used the scaling laws $\chi=\chi_{0} t_{\text {red }}^{-(2-\eta) \nu}$ and $\xi=\xi_{0} t_{\text {red }}^{-\nu}$ for the osmotic susceptibility $\chi$ and correlation length $\xi$, respectively. The critical exponent $\eta$ denotes the Fisher exponent. We limited our measurements to the strong scattering regime to test the one-beam set-up in the vicinity of a critical point. The investigated temperature range was so small, that we only used the simple scaling law approach. This approach ignores the noncritical scattering contributions by the relatively large polymer which have been treated as a constant background $^{23}$ in a previous work or later by Jacobs it et al. ${ }^{25}$ using the more sophisticated crossover approach which incorporates a crossover from the asymptotic Ising behavior to the critical mean-field behavior. The scattering vector $q$ varies in the range of $q=9.70 \times 10^{-3}-2.67 \times 10^{-2} \mathrm{~nm}^{-1}$. The constant $A$ depends on the properties of the sample and the geometrical constraints of the optical set-up, and can be treated as temperature independent.

In order to obtain the singly scattered light intensity, which can be described by Eq. (3), the count rate $\left\langle I_{\text {tot }, \theta}^{\prime}\right\rangle$ measured by the photomultiplier has been corrected for the loss due to turbidity $\tau$ and for multiple scattering $\beta$,

$$
\left\langle i_{s, \theta}\right\rangle=\frac{\left\langle I_{\mathrm{tot}, \theta}^{\prime}\right\rangle \beta}{e^{-\tau l}} \sin (\theta)=\frac{\left\langle I_{\mathrm{tot}, \theta}\right\rangle \beta}{e^{-\tau l}} .
$$

The turbidity $\tau=-\ln (\mathcal{T}) / l$ was calculated from the measured transmission $\mathcal{T}$ and the path length $l$. The term $\sin (\theta)$ accounts for the change in the size of the scattering volume.

The data where fitted to Eq. (3) using the MarquardtLevenberg algorithm ${ }^{26}$ weighting the intensities by their uncertainty. The uncertainty of the intensity is propagated from the uncertainties of the directly measured quantities as follows:

$$
\begin{aligned}
\frac{\sigma_{\left\langle i_{s, \theta}\right\rangle}}{\left\langle i_{s, \theta}\right\rangle}= & {\left[\left(\frac{\partial\left\langle i_{s, \theta}\right\rangle}{\partial T} \frac{\delta T}{\left\langle i_{s, \theta}\right\rangle}\right)^{2}+\left(\frac{\partial\left\langle i_{s, \theta}\right\rangle}{\partial T_{c}} \frac{\delta T_{c}}{\left\langle i_{s, \theta}\right\rangle}\right)^{2}\right.} \\
& \left.+\left(\frac{\delta\left\langle I_{\text {tot }, \theta}\right\rangle}{\left\langle I_{\text {tot }, \theta}\right\rangle}\right)^{2}+\left(\frac{\delta \beta}{\beta}\right)^{2}+(l \delta \tau)^{2}\right]^{1 / 2} .
\end{aligned}
$$

Equation (5) includes temperature fluctuations of $\delta T$ $=2 \mathrm{mK}$ and uncertainties of the critical temperature $\delta T_{c}$ $=2 \mathrm{mK} . \delta\left\langle I_{\text {tot }, \theta}\right\rangle$ is the uncertainty of the total scattering intensity at the scattering angle $\theta$ at constant temperature. $\delta \beta$ is the uncertainty of the amplitude of the cross correlation function calculated as one standard deviation of three measure- 


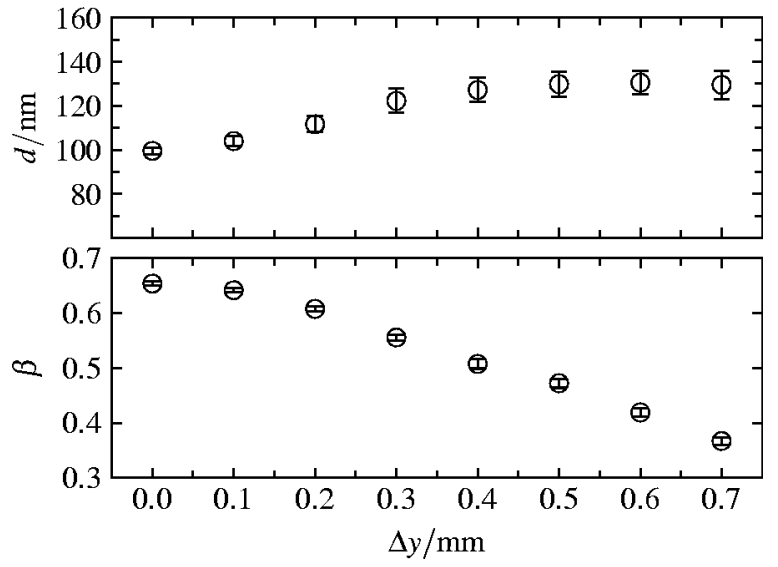

FIG. 1. Alignment of the fibers.

ments. The calculated error of the turbidity $\delta \tau=\delta \mathcal{T}(l \mathcal{T})$ depends on the uncertainty of the transmission $\delta \mathcal{T}$. The uncertainty of the path length was neglected. The uncertainty of the transmission $\mathcal{T}$ was calculated as difference of the maximal and minimal transmission measurement over the entire measurement time. For comparison we performed also the analysis using one standard deviation of the mean, which was calculated from 120 single measurements. Using this error in the transmission seem to underestimate the real error due to the high number of data points resulting in a systematically to high $\chi^{2}$ around 8 . Nevertheless, that change is weighting changed the adjusted parameters only within their error bars.

\section{EXPERIMENT}

\section{A. Sample}

We used polystyrene with a molecular weight $M_{w}$ $=1.11 \times 10^{5} \mathrm{~g} \mathrm{~mol}^{-1}$ and $M_{w} / M_{n}=1.061$, where $M_{w}$ is the weight-average molecular weight and $M_{w} / M_{n}$ is the ratio of weight-to-number-averaged molecular weights. The mixture of polystyrene/cyclohexane has a critical mass fraction of 0.118 and an upper critical point at the critical temperature $T_{c}=293.321 \pm 0.002 \mathrm{~K}$. Our scattering cell used, has an inner diameter of $l=1 \mathrm{~cm}$. In this paper we used the same sample, which was already investigated in Ref. 23. Details of sample preparation are described there.

\section{B. Set-up and temperature control}

The optical set-up of the one-beam technique is very similar to a conventional light scattering experiment, only the detection part is modified. A detailed description of the set-up can be found in Refs. 17 and 22. The scattered light is divided into two beams by a beamsplitter. Two monomode fibers each with an integrated gradient-index lens are used to detect the scattered light. The two fibers can be moved to find the optimal edge of the coherence area.

For the optimal alignment of the fibers we used a concentrated latex suspension $(c=0.1 \mathrm{wt} . \%)$ with a particle diameter of $132 \mathrm{~nm}$. In Fig. 1 we show the particle diameter (d) determined by dynamic light scattering and the ampli-

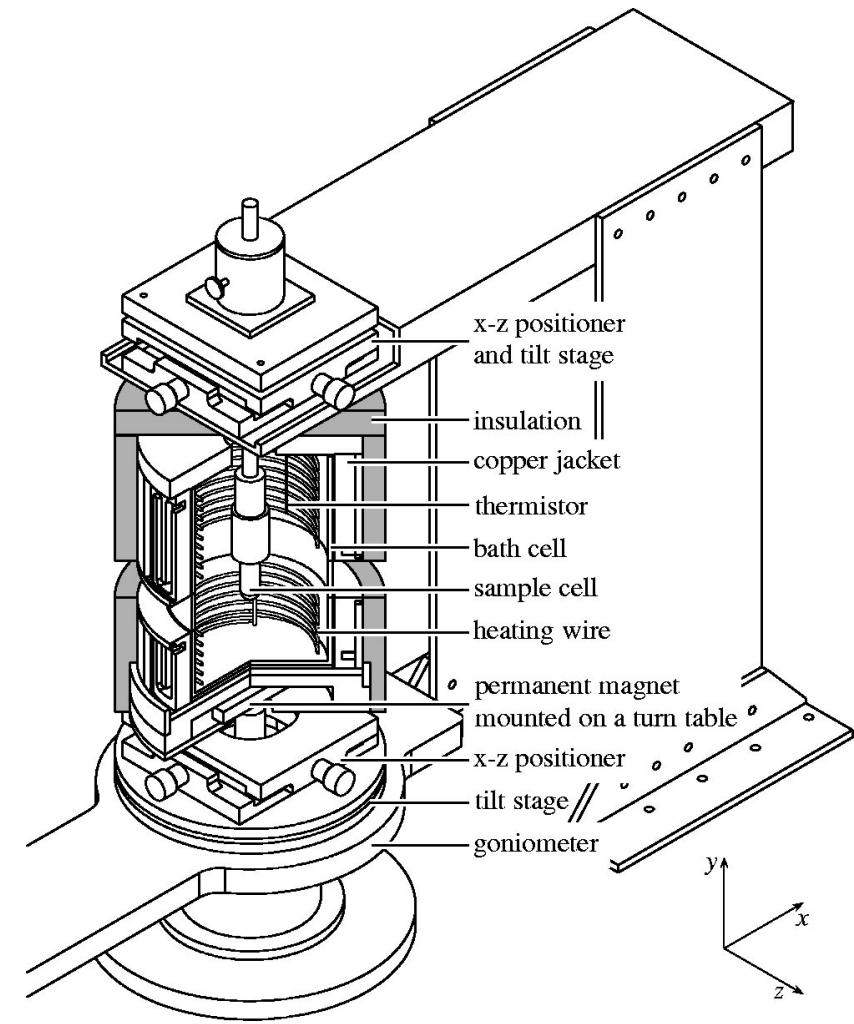

FIG. 2. A sketch of the temperature cell.

tude of cross correlation function $(\beta)$ in dependence of the displacement of the fibers $(\Delta y)$. If the distance between the two fibers vanishes the cross correlation function is equal to the autocorrelation function and contains contributions stemming from multiple scattered light and resulting in a particle diameter which is too small. With increasing distance between the two fibers the multiple scattered light is not longer correlated and the correct particle diameter is obtained.

To achieve a temperature stability of $\delta T=2 \mathrm{mK}$ we designed a cell which is presented in Fig. 2. The sample is immersed in a glass cylinder with a diameter of $110.2 \mathrm{~mm}$. This bath cell was filled with water, which was stirred to avoid temperature gradients. To heat the water bath we use a CuNi-wire. For temperature measurement there is a thermistor (Pewatron, MBT 1.3) with an absolute accuracy of $0.05 \mathrm{~K}$ and a differentially precision better than $1 \mathrm{mK}$. The bath cell is surrounded by a copper cylinder which is moderated with a thermostat (Lauda, RC6). For isolation we use styrodur with a thickness of $1.5 \mathrm{~cm}$. To make a measurement at a certain temperature, the copper cylinder is kept at a temperature which lies $2 \mathrm{~K}$ under the required temperature. The electrical heating is regulated by a computer to achieve the temperature. The measurement of the correlation function was repeated three times, whereby each correlation function was averaged for $60 \mathrm{~min}$ and analyzed separately. The average amplitude $\beta$ is the mean of the three measurements was used to determine the singly scattered intensity $\left\langle i_{s}\right\rangle$ listed in Table I. 
TABLE I. Scattering intensities and corrected scattering intensities for different temperatures and different angles $\theta=40^{\circ}, 60^{\circ}, 80^{\circ}, 90^{\circ}, 100^{\circ}, 120^{\circ}, 140^{\circ}$. The critical temperature is $T_{c}=293.321 \mathrm{~K}$.

\begin{tabular}{|c|c|c|c|c|c|c|c|}
\hline$T / \mathrm{K}$ & $\left\langle I_{\text {tot }, 40}\right\rangle$ & $\left\langle i_{\mathrm{tot}, 40}\right\rangle$ & $\left\langle i_{s, 40}\right\rangle$ & $\mathrm{T} / \mathrm{K}$ & $\left\langle I_{\mathrm{tot}, 90}\right\rangle$ & $\left\langle i_{\text {tot }, 90}\right\rangle$ & $\left\langle i_{s, 90}\right\rangle$ \\
\hline 303.150 & 8.1 & 8.1 & 7.9 & 293.565 & 160.5 & 268.3 & 254.2 \\
\hline 299.526 & 12.1 & 12.3 & 11.4 & 293.473 & 169.0 & 347.1 & 301.7 \\
\hline 297.238 & 19.4 & 19.5 & 18.1 & 293.415 & 165.7 & 428.7 & 342.1 \\
\hline 295.792 & 31.9 & 32.2 & 30.5 & 293.379 & 155.6 & 516.8 & 363.8 \\
\hline 294.880 & 52.6 & 54.9 & 52.1 & 293.356 & 144.4 & 624.4 & 365.7 \\
\hline 294.303 & 85.8 & 95.1 & 90.0 & 293.341 & 130.2 & 738.8 & 342.6 \\
\hline 293.940 & 134.1 & 163.9 & 154.3 & 293.332 & 128.6 & 967.8 & 362.9 \\
\hline 293.710 & 196.9 & 276.1 & 259.5 & 293.326 & 117.4 & 1162.0 & 328.7 \\
\hline 293.565 & 267.8 & 446.3 & 422.7 & \multirow[b]{2}{*}{$T / \mathrm{K}$} & \multirow{2}{*}{$\left\langle I_{\text {tot, }, 100}\right\rangle$} & \multirow[b]{2}{*}{$\left\langle i_{\text {tot }, 100}\right\rangle$} & \multirow[b]{2}{*}{$\left\langle i_{s, 100}\right\rangle$} \\
\hline 293.473 & 333.9 & 682.1 & 574.3 & & & & \\
\hline 293.415 & 378.2 & 968.9 & 702.1 & 303.150 & 6.7 & 6.7 & 6.3 \\
\hline 293.379 & 394.7 & 1289.7 & 1025.4 & 299.526 & 10.9 & 11.1 & 10.0 \\
\hline 293.356 & 389.2 & 1637.6 & 1086.0 & 297.238 & 18.0 & 18.1 & 16.3 \\
\hline 293.341 & 375.7 & 2041.6 & 1192.7 & 295.792 & 29.5 & 29.8 & 28.1 \\
\hline 293.332 & 362.1 & 2542.3 & 1243.3 & 294.880 & 47.0 & 49.0 & 47.2 \\
\hline 293.326 & 361.0 & 3242.9 & 1563.4 & 294.303 & 71.8 & 79.5 & 77.7 \\
\hline \multirow{2}{*}{$T / \mathrm{K}$} & \multirow{2}{*}{$\left\langle I_{\text {tot, } 60}\right\rangle$} & \multirow{2}{*}{$\left\langle i_{\text {tot }, 60}\right\rangle$} & \multirow{2}{*}{$\left\langle i_{s, 60}\right\rangle$} & 293.940 & 101.0 & 123.5 & 120.3 \\
\hline & & & & 293.710 & 128.7 & 180.4 & 170.5 \\
\hline 303.150 & 7.2 & 7.2 & 6.4 & 293.565 & 146.3 & 243.8 & 223.5 \\
\hline 299.526 & 11.6 & 11.8 & 11.0 & 293.473 & 151.3 & 309.0 & 264.3 \\
\hline 297.238 & 19.1 & 19.3 & 18.0 & 293.415 & 145.3 & 372.3 & 292.0 \\
\hline 295.792 & 31.8 & 32.1 & 30.1 & 293.379 & 133.6 & 436.5 & 310.3 \\
\hline 294.880 & 52.0 & 54.3 & 50.6 & 293.356 & 121.2 & 509.8 & 304.2 \\
\hline 294.303 & 83.3 & 92.3 & 85.5 & 293.341 & 110.3 & 599.7 & 291.6 \\
\hline 293.940 & 125.6 & 153.5 & 140.6 & 293.332 & 103.5 & 726.4 & 291.4 \\
\hline 293.710 & 175.5 & 246.0 & 222.1 & 293.326 & 100.8 & 905.3 & 270.1 \\
\hline 293.565 & 221.5 & 369.2 & 333.6 & \multirow{2}{*}{$T / \mathrm{K}$} & \multirow{2}{*}{$\left\langle I_{\text {tot, }, 120}\right\rangle$} & \multirow{2}{*}{$\left\langle i_{\text {tot, } 120}\right\rangle$} & \multirow{2}{*}{$\left\langle i_{s, 120}\right\rangle$} \\
\hline 293.473 & 254.0 & 518.9 & 441.5 & & & & \\
\hline 293.415 & 263.6 & 675.4 & 552.4 & 303.150 & 6.7 & 6.7 & 6.2 \\
\hline 293.379 & 256.4 & 838.0 & 587.8 & 299.526 & 10.9 & 11.1 & 9.9 \\
\hline 293.356 & 241.0 & 1013.9 & 642.0 & 297.238 & 17.9 & 18.0 & 16.6 \\
\hline 293.341 & 224.3 & 1219.1 & 498.4 & 295.792 & 29.1 & 29.3 & 28.1 \\
\hline 293.332 & 212.4 & 1491.3 & 623.0 & 294.880 & 45.8 & 47.8 & 46.6 \\
\hline 293.326 & 207.6 & 1864.9 & 417.6 & 294.303 & 68.7 & 76.1 & 73.9 \\
\hline \multirow{2}{*}{$T / \mathrm{K}$} & \multirow{2}{*}{$\left\langle I_{\text {tot }, 80}\right\rangle$} & $\left\langle i_{-00}\right\rangle$ & $\left\langle i i_{0}\right\rangle$ & 293.940 & 94.1 & 115.1 & 110.4 \\
\hline & & $\left\langle l_{\text {tot }, 80\rangle}\right.$ & $\mid\left\langle l_{s, 80\rangle}\right.$ & 293.710 & 116.3 & 163.0 & 153.1 \\
\hline 303.150 & 6.8 & 6.8 & 6.4 & 293.565 & 128.2 & 213.7 & 195.9 \\
\hline 299.526 & 11.0 & 11.2 & 10.2 & 293.473 & 128.9 & 263.2 & 224.3 \\
\hline 297.238 & 18.1 & 18.3 & 17.1 & 293.415 & 121.1 & 310.3 & 239.7 \\
\hline 295.792 & 30.0 & 30.3 & 29.8 & 293.379 & 109.1 & 356.4 & 237.3 \\
\hline 294.880 & 48.7 & 50.8 & 50.5 & 293.356 & 97.5 & 410.1 & 239.7 \\
\hline 294.303 & 76.5 & 84.8 & 84.8 & 293.341 & 87.5 & 475.3 & 220.8 \\
\hline 293.940 & 111.5 & 136.2 & 134.3 & 293.332 & 81.7 & 573.4 & 246.6 \\
\hline 293.710 & 148.5 & 208.1 & 201.9 & 293.326 & 79.1 & 710.6 & 237.3 \\
\hline 293.565 & 177.0 & 295.0 & 272.2 & & & & \\
\hline 293.473 & 190.9 & 390.0 & 344.1 & $T / \mathrm{K}$ & $\left\langle I_{\text {tot }, 140}\right\rangle$ & $\left\langle i_{\text {tot }, 140}\right\rangle$ & $\left\langle i_{s, 140}\right\rangle$ \\
\hline 293.415 & 189.2 & 484.7 & 412.6 & 303.150 & 6.8 & 6.8 & 6.1 \\
\hline 293.379 & 177.9 & 581.3 & 384.2 & 299.526 & 10.7 & 10.9 & 10.0 \\
\hline 293.356 & 163.8 & 689.1 & 389.7 & 297.238 & 17.5 & 17.6 & 16.4 \\
\hline 293.341 & 150.8 & 819.4 & 385.8 & 295.792 & 28.5 & 28.8 & 27.1 \\
\hline 293.332 & 141.4 & 993.0 & 366.4 & 294.880 & 44.7 & 46.6 & 44.2 \\
\hline 293.326 & 138.1 & 1240.5 & 435.7 & 294.303 & 66.2 & 73.4 & 69.4 \\
\hline$T / \mathrm{K}$ & $\left\langle I_{\text {tot }, 90}\right\rangle$ & $\left\langle i_{\text {tot } 90}\right\rangle$ & $\left\langle i_{s, 90}\right\rangle$ & 293.940 & 89.3 & 109.2 & 101.8 \\
\hline $1 / \mathrm{N}$ & $\left\langle I_{\text {tot }, 90\rangle}\right\rangle$ & $\left\langle l_{\text {tot }, 90\rangle}\right\rangle$ & $\left\langle l_{s, 90\rangle}\right.$ & 293.710 & 107.7 & 150.9 & 137.0 \\
\hline 303.150 & 6.7 & 6.7 & 6.2 & 293.565 & 116.1 & 193.6 & 170.3 \\
\hline 299.526 & 10.9 & 11.1 & 10.3 & 293.473 & 114.4 & 233.7 & 193.5 \\
\hline 297.238 & 17.8 & 18.0 & 17.1 & 293.415 & 105.6 & 270.5 & 205.2 \\
\hline 295.792 & 29.4 & 29.7 & 28.8 & 293.379 & 93.4 & 305.3 & 206.2 \\
\hline 294.880 & 47.5 & 49.5 & 48.8 & 293.356 & 81.9 & 344.5 & 199.9 \\
\hline 294.303 & 73.4 & 81.4 & 81.0 & 293.341 & 72.7 & 395.1 & 198.1 \\
\hline 293.940 & 104.4 & 127.7 & 127.7 & 293.332 & 66.7 & 468.6 & 197.4 \\
\hline 293.710 & 137.6 & 193.3 & 186.4 & 293.326 & 64.1 & 576.2 & 193.4 \\
\hline
\end{tabular}




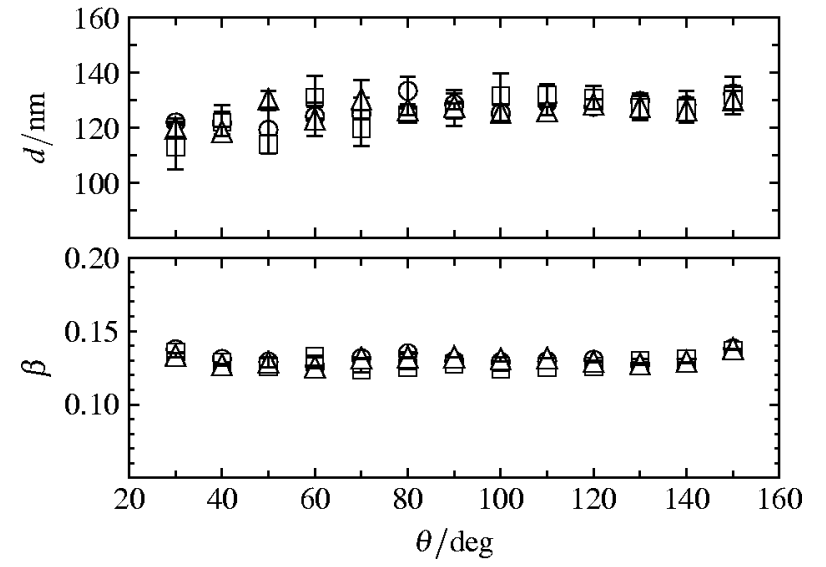

FIG. 3. Angular dependence of amplitude of cross correlation function for different temperatures. $\bigcirc: 20^{\circ} \mathrm{C}, \square, 25^{\circ} \mathrm{C}, \triangle, 30^{\circ} \mathrm{C}$.

\section{Influence of temperature changes to the alignment}

First we tested the set-up for temperature dependent measurements with standard polystyrene latex spheres. It is known that the refractive index changes with temperature and therefore the position of the focus of the incident laser beam will change with the temperature. This will also change the size of the speckles on the detector and might make a realignment was necessary. In order to check whether a realignment would be necessary we did some preliminary measurements. In Fig. 3 we show the angular dependence of particle diameter $(d)$ determined by dynamic light scattering and the amplitude of cross correlation function $(\beta)$ for different temperatures. Neither the particle diameter nor the amplitude of the cross correlation function show a temperature dependence in the investigated temperature range of $20-30{ }^{\circ} \mathrm{C}$. Therefore, we conclude that the temperature variation of the refractive index in this region causes no noticeable change of size and position of the scattering volume in our set-up. This fact enables us to compare the data at different temperatures.

The mean value of the diameter is $\bar{d}=126 \pm 4 \mathrm{~nm}$, whereas it was determined by averaging over all angles and temperatures. If one compares the average diameter $\bar{d}_{30^{\circ}-50^{\circ}}=120 \pm 2 \mathrm{~nm}$ for the three angles below $60^{\circ}$ and the average diameter $\bar{d}_{60^{\circ}-150^{\circ}}=128 \pm 2 \mathrm{~nm}$ for the angles above $60^{\circ}$, one finds a systematic decrease of the diameter by $6.5 \%$ at the lower scattering angles. This might be an indication for an incomplete suppression of the multiply scattered light at low scattering angles or the presence of interactions at higher concentrations. Comparison with measurements of aqueous solutions of latex particles with a diameter of $d=80 \mathrm{~nm}$ do not show this systematic decrease of the determined diameter at small angles. A comparison with larger particles reveals at higher concentration a clear influence of interactions, which has also been demonstrated with the $3 \mathrm{D}$ cross correlation technique. ${ }^{12}$ Analyzing the static properties of Latex particles with a diameter of $d=453 \mathrm{~nm}$ (Ref. 17) we observed a good agreement between the different concentrations over the entire investigated concentration and angle range. Due to the fact that it is difficult to separate the influence on the decay constant due to interaction or insufficient suppression of

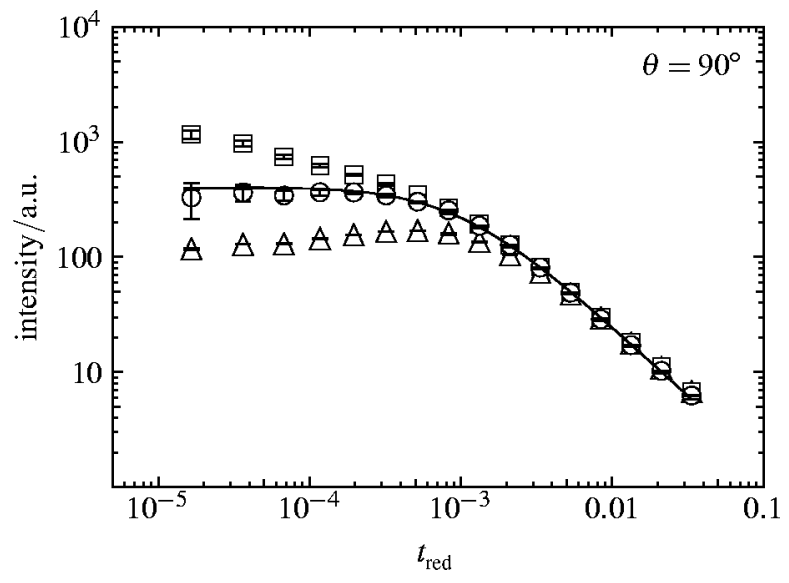

FIG. 4. Scattering intensity $\left\langle I_{\text {tot,90 }}\right\rangle(\triangle)$, intensity corrected for turbidity loss $\left\langle i_{\text {tot,90 }}\right\rangle(\square)$ and intensity corrected for turbidity loss and multiple scattering $\left\langle i_{s, 90}\right\rangle(\bigcirc)$ in dependence on the reduced temperature at $\theta=90^{\circ}$. The solid line refers to fit No. 4 in Table II.

multiple scattering we perform the latter analysis of the critical data including the small scattering angle and neglecting them to see whether a possible insufficient suppression is possible at smaller scattering angles.

\section{EXPERIMENTAL RESULTS AND DISCUSSION}

In Table I we summarize the dependence of the scattering intensities $\left\langle I_{\text {tot }, \theta}\right\rangle$, intensities corrected for turbidity loss $\left\langle i_{\text {tot }, \theta}\right\rangle=\left\langle I_{\text {tot }, \theta}\right\rangle / e^{-\tau l}$ and intensities corrected for turbidity loss and multiple scattering $\left\langle i_{s, \theta}\right\rangle=\left\langle I_{\mathrm{tot}, \theta}\right\rangle / e^{-\tau l}$ on the temperature $T$ for seven different angles $\theta=40^{\circ}, 60^{\circ}, 80^{\circ}, 90^{\circ}$, $100^{\circ}, 120^{\circ}, 140^{\circ}$.

The scattering intensity at $\theta=90^{\circ}$ as a function of reduced temperature is shown in Fig. 4 (other angles in Fig. 5). The three curves show the total scattering intensity $\left\langle I_{\text {tot, } 90}\right\rangle$ $(\triangle)$, the intensity corrected for turbidity loss $\left\langle i_{\text {tot,90 }}\right\rangle(\square)$ and intensity corrected for turbidity loss and multiple scattering $\left\langle i_{s, 90}\right\rangle(\bigcirc)$. The solid line refers to a fit according to Eq. (3) with $\nu=0.63$ fixed to its theoretical value with the parameter set listed in row 4 in Table II. The Fisher exponent is fixed to its theoretical value of $\eta=0.032$. At reduced temperatures up to $10^{-2}$ where the turbidity level and multiple scattering contributions are small, all curves agree. The data of $\left\langle I_{\text {tot }, 90}\right\rangle$ passes through a pronounced maximum and cannot be described by the Ornstein-Zernike equation [see Eq. (3)]. A description of the turbidity corrected data by the OrnsteinZernike equation leads to systematic deviations close to the critical point. Applying both the turbidity correction and the multiple scattering correction obtained from the amplitudes of the cross correlation function, the data can be described by the Ornstein-Zernike equation over the entire temperature range. Treating $\nu$ as an adjustable parameter does not improve the result significantly (Table II, set \#11).

The parameters obtained for all angles with the critical exponent $\nu$ both fixed and as adjustable parameter are listed in Table II. Allowing $\nu$ to be an adjustable parameter leads in average of all angles to a mean critical exponent of $\bar{\nu}$ $=0.63 \pm 0.01$ which agrees well with the theoretically expected value. The average correlation length amplitudes $\bar{\xi}_{0}$ 

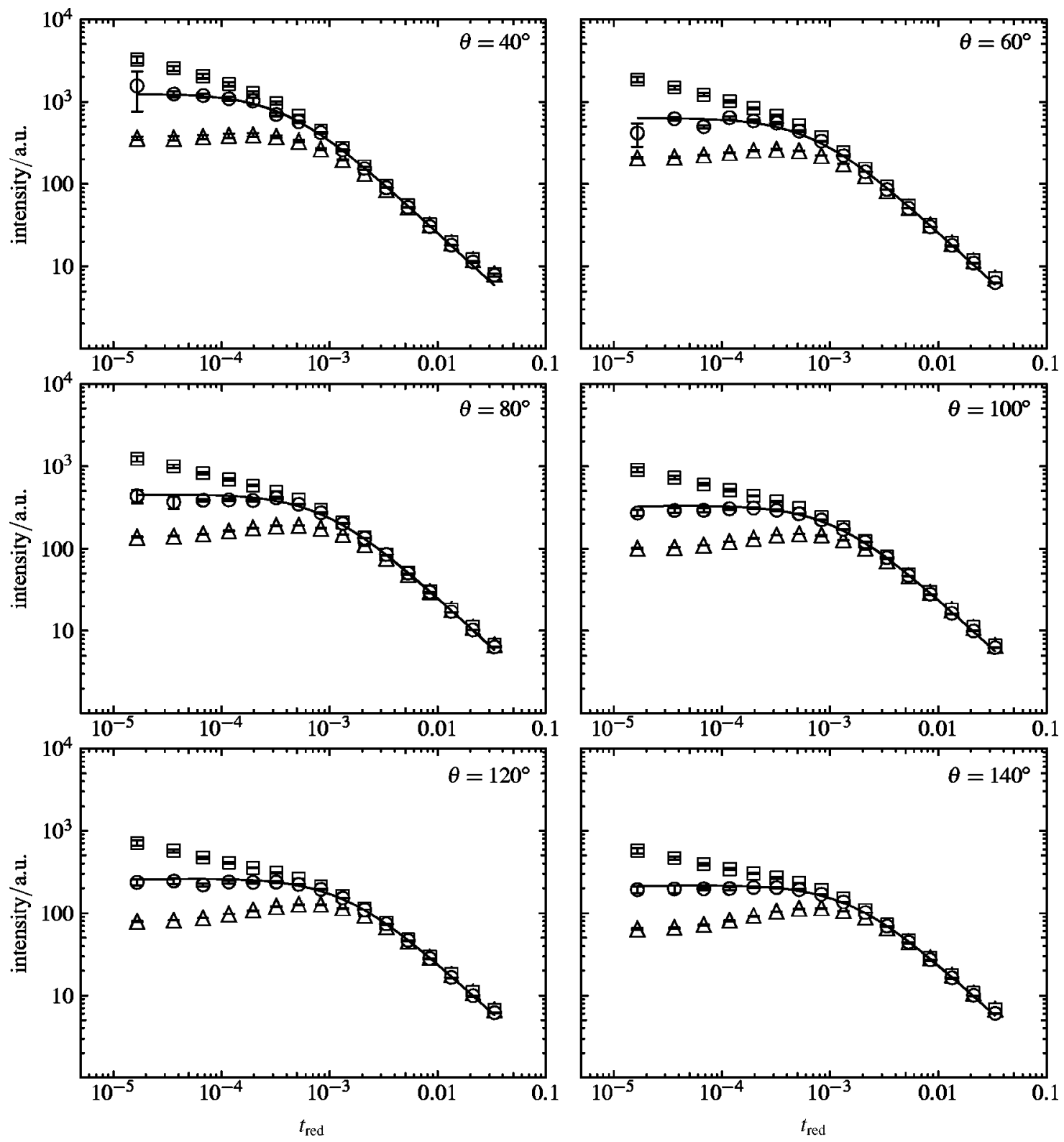

FIG. 5. Scattering intensity $\left\langle I_{\text {tot }, \theta}\right\rangle(\triangle)$, intensity corrected for turbidity loss $\left\langle i_{\text {tot }, \theta}\right\rangle(\square)$ and intensity corrected for turbidity loss and multiple scattering $\left\langle i_{s, \theta}\right\rangle$ $(\bigcirc)$ in dependence on the reduced temperature for six different angles $\theta=40^{\circ}, 60^{\circ}, 80^{\circ}, 100^{\circ}, 120^{\circ}, 140^{\circ}$. The solid lines represent the fits according to Eq. (3) with $\nu=0.63$ fixed to its theoretical value.

$=0.69 \pm 0.04 \mathrm{~nm}(\nu$ fixed $)$ and $\bar{\xi}_{0}=0.70 \pm 0.07 \mathrm{~nm}(\nu$ adjustable) agree within their error bars. The simultaneous fit of all angles at fixed $\nu$ by minimizing the sum of the $\chi^{2}$ leads to a correlation length amplitude of $\bar{\xi}_{0}=0.68 \mathrm{~nm}$ which agrees well will the average value of $\bar{\xi}_{0}$. It is obvious, that the two lower angles lead to systematically higher values of $\xi_{0}$. Omitting those results in $\bar{\xi}_{0}=0.67 \pm 0.01 \mathrm{~nm}$ ( $\nu$ fixed) and $\bar{\xi}_{0}=0.66 \pm 0.01 \mathrm{~nm}$ ( $\nu$ adjustable). Ritzl and Woermann derived a power law $\xi_{0}=\xi_{0}^{*}\left(M_{n} / M_{0}\right)^{n}$ for the correlation length amplitude of a critical polystyrene/cyclohexane mixture in dependence of the number-weighted molar mass. ${ }^{27}$ $M_{0}$ is the mass of the repeat unit and $\xi_{0}^{*}=0.149 \mathrm{~nm}$ and $n$ $=0.218$ are two empirical scaling parameters. For our system this leads to $\xi_{0}=0.67 \mathrm{~nm}$, which agrees well with the above values. Nevertheless, in our previous work we obtain a
TABLE II. Experimental results of the Ornstein-Zernike fits for different angles.

\begin{tabular}{rrcccc}
\hline \hline No. & $\theta /^{\circ}$ & $\nu$ & $\xi_{0} / \mathrm{nm}$ & $A / 10^{-2}$ & $\chi^{2}$ \\
\hline 1 & 40 & 0.630 & $0.767 \pm 0.017$ & $8.43 \pm 0.09$ & 2.5 \\
2 & 60 & 0.630 & $0.748 \pm 0.010$ & $8.62 \pm 0.07$ & 4.3 \\
3 & 80 & 0.630 & $0.685 \pm 0.008$ & $8.49 \pm 0.06$ & 3.4 \\
4 & 90 & 0.630 & $0.658 \pm 0.007$ & $8.41 \pm 0.06$ & 1.8 \\
5 & 100 & 0.630 & $0.664 \pm 0.007$ & $8.26 \pm 0.06$ & 3.8 \\
6 & 120 & 0.630 & $0.666 \pm 0.007$ & $8.39 \pm 0.06$ & 2.7 \\
7 & 140 & 0.630 & $0.668 \pm 0.006$ & $8.27 \pm 0.06$ & 2.3 \\
8 & 40 & $0.612 \pm 0.007$ & $0.813 \pm 0.026$ & $10.07 \pm 0.71$ & 2.2 \\
9 & 60 & $0.618 \pm 0.005$ & $0.778 \pm 0.017$ & $9.60 \pm 0.44$ & 4.2 \\
10 & 80 & $0.643 \pm 0.005$ & $0.654 \pm 0.014$ & $7.50 \pm 0.35$ & 3.1 \\
11 & 90 & $0.631 \pm 0.005$ & $0.655 \pm 0.012$ & $8.33 \pm 0.37$ & 2.0 \\
12 & 100 & $0.629 \pm 0.005$ & $0.666 \pm 0.013$ & $8.31 \pm 0.37$ & 4.0 \\
13 & 120 & $0.633 \pm 0.005$ & $0.658 \pm 0.013$ & $8.13 \pm 0.37$ & 2.9 \\
14 & 140 & $0.624 \pm 0.005$ & $0.684 \pm 0.014$ & $8.76 \pm 0.42$ & 2.4 \\
\hline \hline
\end{tabular}




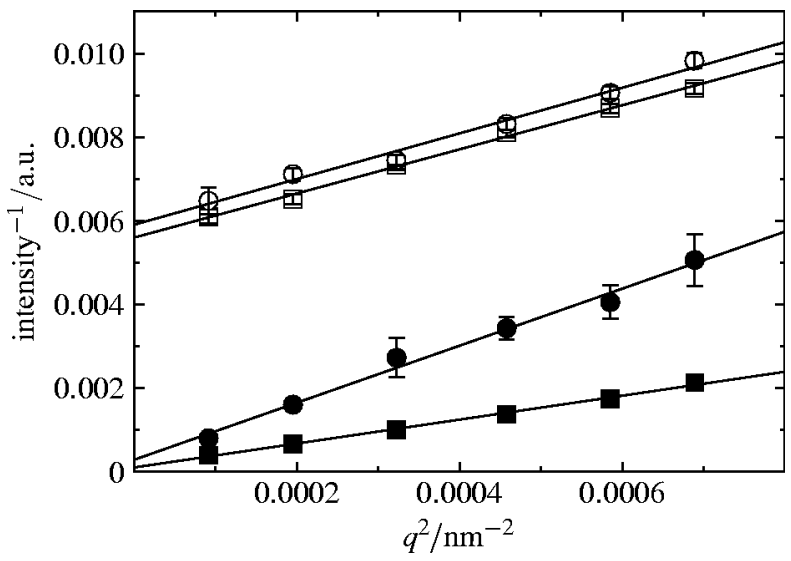

FIG. 6. Angular dependence of scattered intensity for two different reduced temperatures (open symbols, $t_{\text {red }}=2.11 \times 10^{-3}$; solid symbols, $t_{\text {red }}=3.63$ $\times 10^{-5}$ ). The squares represent intensities corrected for turbidity loss $\left\langle i_{\text {tot }}\right\rangle$ and the circles represent intensities corrected for turbidity loss and multiple scattering $\left\langle i_{s}\right\rangle$.

correlation length amplitude of $\xi_{0}=0.62 \pm 0.01 \mathrm{~nm}$ which is slightly lower. ${ }^{23}$ Recent measurements of the angular dependent scattering intensity on the same sample in the 3D set-up lead also to a slightly higher correlation length amplitude of $\xi_{0}=0.66 \mathrm{~nm}$ (Ref. 28) which agrees well with the present work. The discrepancy of the lower correlation length amplitude in previous and actual measurement might be caused by aging of the sample which did not influence the critical temperature $^{32}$ but the turbidity. A change in the turbidity level due to aging of the sample has also been observed before. ${ }^{29}$ The turbidity change resulted in an increase of the correlation length amplitude, while the critical temperature remained almost unchanged (less than $2.5 \mathrm{mK} /$ week). In conclusion, one can state, that all measurements are consistent over the entire temperature and angle range and can be analyzed using the Ornstein-Zernike equation [cf. Eq. (3)] and it seems to be reasonable to exclude the two lower angles and give an average value of the correlation length amplitude of $\bar{\xi}_{0}=0.66 \pm 0.02 \mathrm{~nm}$.

In Fig. 6 we show the inverse scattering light intensity as a function of squared wave vector $q^{2}$ (Ornstein-Zernike plot) at two different temperatures. The squares represent intensities corrected for turbidity loss $\left\langle i_{\text {tot }}\right\rangle$ and the circles represent intensities corrected for turbidity loss and multiple scattering $\left\langle i_{s}\right\rangle$. All data show a linear dependence of the square of the scattering vector $q^{2}$ as it is expected according to Eq. (3). In the vicinity of the critical point the slope and the intercept of the uncorrected data (squares) change strongly compared to the corrected data.

Figure 7 shows the correlations length $\xi$ determined by the Ornstein-Zernike plot in dependence of the reduced temperature. The solid line corresponds to an asymptotic power law $\xi=\xi_{0} t_{\text {red }}^{-\nu}$, with $\xi_{0}=0.61 \pm 0.02 \mathrm{~nm}$ and $\nu=0.63$. There slight systematic deviations from the power law behavior far away from the critical point, which might be an indication for crossover behavior, which is not visible in the dependence of the scattering intensity of the reduced temperature. Far away from the critical point the angular dependence of the scattering intensity as indicated also by the large error

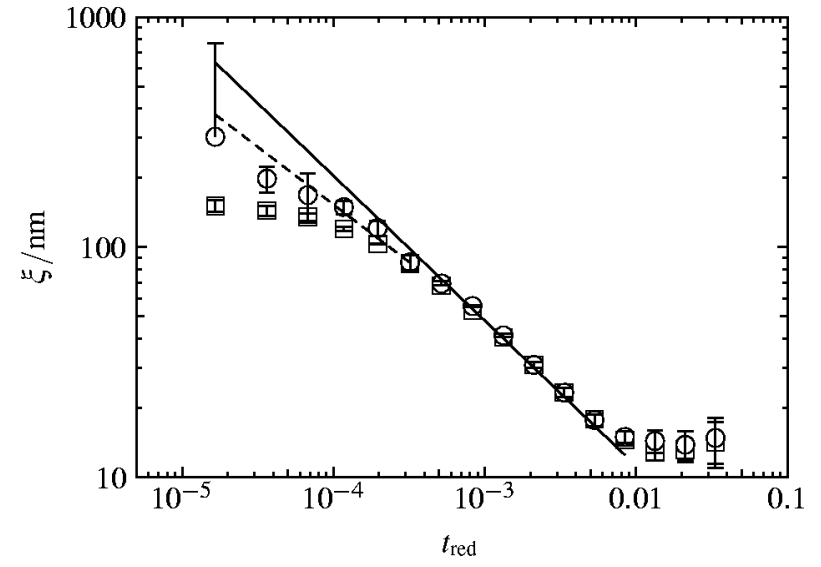

FIG. 7. Correlation length $\xi$ determined by angular dependent measurements of static scattering intensity. The squares represent intensities corrected for turbidity loss $\left\langle i_{\text {tot }}\right\rangle$ and the circles represent intensities corrected for turbidity loss and multiple scattering $\left\langle i_{s}\right\rangle$. The solid line represents a critical exponent $\nu=0.63$ while $\nu=0.5$ for the dashed line.

bars is not very pronounced, which easily leads to a falsification of the results. Close to the critical point strong deviations from the asymptotic power law behavior are observed in both the uncorrected and the corrected data. Similar deviations were not observed in the analysis of the scattering intensities dependent on the reduced temperature for a fixed scattering angle. So it is possible that the deviations are caused by numerical instabilities due to the small intercept which leads to large uncertainties close to the critical point. Furthermore the deviations could stem from insufficient suppression of the multiple scattered light in the one-beam set-up as might also indicated by the low scattering angles in the angular dependence of the diameter (cf. Sec. IIIC). Therefore, we perform the analysis also without the $60^{\circ}$ scattering angle, but the result remained the same. Additional measurements on the same sample in a 3D set-up ${ }^{28}$ showed also systematic deviations from the scaling law behavior at a reduced temperature just below $t_{\text {red }}=10^{-4}$. The multiple suppression principles are quite different in both set-ups and both methods work successfully at higher turbidity levels as was shown by the investigation of highly turbid Latex suspensions. ${ }^{12,17}$ Therefore, it is unlikely that the deviations stem from a breakdown of the one-beam set-up. To resolve this open question measurements with the two-color set-up would also be desirable. Deviations from the asymptotic behavior of the isothermic compressibility were also reported by Wagner and co-workers ${ }^{30,31}$ in pure sulfur hexafluoride and pure carbon dioxide very close to the critical point. The exponents found were slightly smaller than the classical exponents. They discussed the possibility that due to the gravitational field deviations from the 3D-Ising renormalization value could be expected, which has not been proven yet by renormalization theories. The dashed line in Fig. 7 shows that our data agree also with a classical exponent within the error bars, but a similar explanation as in the one-component mixtures is unlikely because binary fluid mixtures are less sensitive to gravity effects. Due to the fact that all experimental data points close to the critical point have been influ- 
enced by multiple scattering so the data basis is quite limited to deduce final conclusion.

To answer this open question more critical mixtures, especially with large correlation length amplitudes need be investigated by cross correlation methods. Such systems would have the advantage that the multiple scattering sets in further away from the critical point, where the recorded data have a higher accuracy. For the investigated system the one-beam set-up and the 3D set-up lead to comparable results. Both systems are equally suited to do temperature dependent measurement. The analysis of the angular dependence of the scattering intensity favors slightly the 3D set-up. It would be desirable to have comparative measurements by the two color set-up, which has the highest theoretical amplitude of the cross correlation function. The difficulty here are the temperature dependent measurements which require due to the change in refractive index a realignment of the two incoming beams.

\section{ACKNOWLEDGMENTS}

The authors want to thank Wolffram Schröer, Jenja Sevenard, and Malte Kleemeier for fruitful discussions. We thank Gerhard Wegner for the constant interest in our work. Elizabeth Lupton we thank for reading the paper.

${ }^{1}$ J.V. Sengers and J.M.H. Levelt Sengers, in Progress in Liquid Physics, edited by C. Croxton (Wiley, New York, 1979), p. 103.

${ }^{2}$ M.E. Fisher, Rev. Mod. Phys. 70, 653 (1998).

${ }^{3}$ J.M.H. Levelt Sengers, A.H. Harvey, and S. Wiegand, "Ionic fluids near critical points and at high temperatures," in Equations of State for Fluids and Fluid Mixtures, edited by J.V. Sengers, R.F. Kayser, C.J. Peters, and H.J. White, Jr. (Elsevier, Amsterdam, 2000), Vol. II.

${ }^{4}$ K. Gutkowski, M.A. Anisimov, and J.V. Sengers, J. Chem. Phys. 114, 3133 (2001).

${ }^{5}$ R. Kita, K. Kubota, and T. Dobashi, Phys. Rev. E 58, 793 (1998).
${ }^{6}$ J.G. Shanks and J.V. Sengers, Phys. Rev. A 38, 885 (1988).

${ }^{7}$ J.K.G. Dhont, Physica A 120, 238 (1983).

${ }^{8}$ J.K.G. Dhont, Physica A 129, 374 (1985).

${ }^{9}$ A.E. Bailey and D.S. Cannell, Phys. Rev. E 50, 4853 (1994).

${ }^{10}$ M. Drewel, J. Ahrens, and U. Podschus, J. Opt. Soc. Am. A 7, 206 (1990).

${ }^{11}$ K. Schätzel, M. Drewel, and J. Ahrens, J. Phys.: Condens. Matter 2, SA393 (1990).

${ }^{12}$ L.B. Aberle, P. Hülstede, S. Wiegand, W. Schröer, and W. Staude, Appl. Opt. 37, 6511 (1998).

${ }^{13}$ C. Urban and P. Schurtenberger, J. Colloid Interface Sci. 207, 150 (1998).

${ }^{14}$ E. Overbeck and C. Sinn, J. Mod. Opt. 46, 303 (1999).

${ }^{15}$ W.V. Meyer, D.S. Cannell, A.E. Smart, T.W. Taylor, and P. Tin, Appl. Opt. 36, 7551 (1997).

${ }^{16}$ A. Moussaid and P.N. Pusey, Phys. Rev. E 60, 5670 (1999).

${ }^{17}$ J.-M. Schröder and S. Wiegand, Phys. Chem. Chem. Phys. 2, 1493 (2000).

${ }^{18}$ K. Schätzel, J. Mod. Opt. 38, 1849 (1991).

${ }^{19}$ P.N. Pusey, Curr. Opin. Colloid Interface Sci. 4, 177 (1999).

${ }^{20}$ U. Nobbmann, S.W. Jones, and B.J. Ackerson, Appl. Opt. 36, 7571 (1997).

${ }^{21}$ A.J. Adorjan, J.A. Lock, T.W. Taylor, P. Tin, W.V. Meyer, and A.E. Smart, Appl. Opt. 38, 3409 (1999).

${ }^{22}$ J.-M. Schröder and S. Wiegand, Soft Matter 1, 53 (2003).

${ }^{23}$ J.-M. Schröder, S. Wiegand, L.B. Aberle, M. Kleemeier, and W. Schröer, Phys. Chem. Chem. Phys. 1, 3287 (1999).

${ }^{24}$ R.F. Chang, H. Burstyn, and J.V. Sengers, Phys. Rev. A 19, 866 (1979).

${ }^{25}$ J. Jacob, M.A. Anisimov, J.V. Sengers, V. Dechabo, I.K. Yudin, and R.W. Gammon, Appl. Opt. 40, 4160 (2001).

${ }^{26}$ P.R. Bevington, Data Reduction and Error Analysis for the Physical Sciences (McGraw-Hill, New York, 1969).

${ }^{27}$ A. Ritzl and D. Woermann, Macromolecules 29, 6671 (1996).

${ }^{28}$ E. Sevenard, thesis, University Bremen, 2003.

${ }^{29}$ S. Wiegand, J. M. H. Levelt Sengers, K.J. Zhang, M.E. Briggs, and R.W. Gammon, J. Chem. Phys. 106, 2777 (1997).

${ }^{30}$ W. Wagner, N. Kurzeja, and B. Pieperbeck, Fluid Phase Equilib. 79, 151 (1992).

${ }^{31}$ N. Kurzeja, Th. Tielkes, and W. Wagner, Int. J. Thermophys. 20, 531 (1999).

${ }^{32} \mathrm{In}$ the previous work we determined critical temperature $T_{c}=293.492 \mathrm{~K}$ with an uncalibrated thermistor. At the same time we performed also measurements of the critical temperature with a calibrated thermistor and obtained $T_{c}=293.287 \mathrm{~K}$, which agrees well with critical temperature in the present work. 